\title{
Magnetic field \& neutron stars: A comprehensive study
}

\author{
Ritam Mallick* \\ Indian Institute of Science Education and Research Bhopal, Bhopal 462066, India \\ E-mail: mallick@iserb.ac.in
}

\section{Stefan Schramm}

Frankfurt Institute for Advanced studies, Frankfurt am Main, Germany

\section{Veronica Dexheimer}

Kent State University, Kent, OH 44242, USA

\section{Abhijit Bhattacharyya}

University of Calcutta, Kolkata 700009, India

The extremely dense matter and frequently strong magnetic fields of neutron stars (NS's) affect profoundly their observable manifestations, including thermal and magnetic evolution, rotation dynamics, and seismology. The ultra-strong magnetic fields present in some anomalous X-ray pulsars (AXP) and soft-gamma repeaters (SGR) (usually reffered to as magnetars) can be as high as $10^{14}-10^{15} \mathrm{G}$ on the surface. Simple estimates from the Virial theorem show that the magnetic field at the core of a NS can be of few times $10^{18} \mathrm{G}$. Such strong magnetic fields can play an effective role in determining the structure and the observable signatures of NS's. Numerical calculations of such axisymmetric deformation of NS's with poloidal and toroidal magnetic fields found deformations of the order of few ten of percent. On the other extreme, there are calculations discussing the effect of the magnetic field on the equation of state (EoS) of the neutron stars. In most of these calculations, a density-dependent magnetic field profile with large central field strengths is assumed. In this work, a general 2D technique is employed, where the Einstein equations are solved by epanding the magnetic field and the perturbed metric in terms of spherical harmonics. The maximum deformation is seen to be of few percent which is in agreement with numerical calculations. When the rotation and magnetic axes are not aligned, higher order perturbation terms may contribute significantly, paving the way for strong gravitational wave (GW) signals. The possibility of vector rho-meson condensation in dense matter subject to strong magnetic fields is also briefly discussed in this manuscript.

7th International Conference on Physics and Astrophysics of Quark Gluon Plasma

1-5 February, 2015

Kolkata, India

${ }^{*}$ Speaker. 


\section{Introduction}

One of the most central topics in astrophysics is the study of compact stars. In addition to the importance of the objects themselves, they also serve as a key tool to further the understanding of hadronic and possibly quark matter at very large densities and low temperatures, rendering compact star physics an ideal complementary approach to the study of high-temperature relativistic heavyion collisions. Compact stars (neutron stars (NS's) and hybrid stars (HS's)) frequently also feature extremely strong magnetic fields, which can affect their observable manifestations, including the thermal and magnetic evolution, rotation dynamics, and seismology.

Among recent observations, the crucial discoveries of the 2-solar-mass pulsars PSR J16142230 and PSR J0348+0432 [1,2] have led to a flurry of theoretical activities in describing heavy neutron stars. The new mass requirement puts severe constraints on modeling the equation of state (EoS) of matter at extreme densities. Soft EoS's, which are not able to generate 2 solar mass neutron stars, are now discarded (including many models that lead to large Bose condensates) and so the models with exotic-particles have come under close scrutiny, although not all are discarded. Overall, the observed pulsar masses have narrowed the range of feasible EoS's, requiring a stiff EoS at high densities.

The frequent occurrence of very strong magnetic fields belongs to the most striking features of NS's. The observed magnetic fields of pulsars are of the order of $10^{8}-10^{12} \mathrm{G}$ at the surface. Recent observations of several new pulsars, namely anomalous X-ray pulsars (AXP's) and softgamma repeaters (SGR's), have been identified to have even larger surface magnetic fields. As the SGR's are usually associated with supernova remnants, they are assumed to be young NS's. The measurement of their spin-down and the rate of change of spin-down suggest that they are quite different from the bulk population of pulsars, as their surface magnetic field may be as high as $10^{15} \mathrm{G}$. Some X-ray pulsar observations also suggest that these objects have surface magnetic fields with strength around $10^{14}-10^{15} \mathrm{G}$. Such pulsars with strong magnetic fields are separately termed magnetars $[3,4]$. The origin of these magnetic fields in NS's remains unclear (trapping of magnetic lines during their collapse can barely yield $10^{8}-10^{9} \mathrm{G}$ ), but the observed spin-down rates of pulsars, which are rotating NS, do require such magnetic fields.

Several mechanisms, such as the flux conservation and dynamo mechanism, have been proposed as the origin of strong magnetic fields inside NS's. The similarity of the magnetic flux distribution in main sequence stars and compact objects was pointed out by several authors, which supports the hypothesis of generation of magnetic fluxes earlier in progenitor stars with subsequent trapping inside the compact objects. Nevertheless, the fields inside a main sequence progenitor may not be enough to yield the required magnetic field inside a NS, as it contains only around $15 \%$ or less of the progenitor mass.

On the other hand, magnetic fields may also be generated through dynamo processes in a rotating and convective fluid. It has been shown [3,4] that newly born NS are likely to combine convection and differential rotation making it favorable for dynamo processes to operate. It was also suggested that the generated fields could explain objects like the soft gamma repeaters and anomalous X-ray pulsars. Large-scale magnetic fields are traditionally associated with convective zones. In this case, the field strengths are often estimated by assuming equipartition of magnetic and kinetic energy density of convection. The generation of the magnetic field may be due to differential rotation as well, though it is unlikely to produce a large-scale field. However, overall, the exact form or the evolution of such strong fields inside a NS is still a very important and unresolved problem.

The surface magnetic field of a NS can be calculated, as well as the exterior morphology 
which is observed to be of dipolar nature. However, the field inside the star is still not very well understood, and the evolution of magnetic fields inside the star is highly uncertain. While magnetic fields as high as $10^{14}-10^{15} \mathrm{G}$ have been inferred at the surface of magnetars [5, 6, 7], there is indirect evidence for fields as high as $10^{16} \mathrm{G}$ inside the star [8]. However, the upper bound in the cores of neutron stars is still not well understood. Most of the general relativity calculations $[8,9,10]$ show central magnetic fields lying somewhere between $10^{18}-10^{19} \mathrm{G}$. Similar values are also obtained from virial theorem estimates employing a classical calculation, which was discussed recently $[11,12]$. The exact value of the magnetic field and its structure depend strongly on the region of the star in which the magnetic field is assumed to be found. This limit also needs to be calculated taking into account general relativity and magnetic field modifications in the metric, not to mention magnetic field modifications to the equation of state itself, which also has its central density substantially modified [13].

\section{Deformation due to magnetic field}

If stars with such large magnetic fields exist, the fields must have a non-negligible effect on the metric describing the star. Similar to rotation, the magnetic field also leads to deformation. The deformation of magnetized NS's was first discussed by Chandrasekhar \& Fermi [14]. Within the general relativistic approach by Bocquet et al. [9] and Bonazolla \& Gourgoulhon [15] and Cardall et al. [10], the deformation of a star from poloidal and toroidal magnetic fields was determined. For field strengths of the order of a few times $10^{18} \mathrm{G}$, the deformation invoked by the magnetic field were of the order of several tens of a percent. However, recent magneto-hydrodynamic (MHD) simulations, which study the dynamics and the stability of magnetars, have hinted to the possibility that the magnetic field inside the star could be much more complicated. There, the axisymmetric field is composed of a poloidal field, which extends throughout the star and in the exterior, while the toroidal field remains confined in a torus-shaped region inside the star where the field lines are closed. This configuration is known as "twisted torus". With such configuration [16] and the magnetic field of the order of $10^{16} \mathrm{G}$, the deformation of the star was calculated to be of the order of $10^{-3}$ to $10^{-4}$.

At the other extreme, there are many calculations in the literature, discussing the effect of the magnetic field on the EoS of the neutron star. The effects of strong magnetic fields on dense hadronic matter have been extensively studied [17, 18, 19]. In most of these calculations [18, 20, $21,22]$ a density-dependent magnetic field profile with large central magnetic fields was assumed. Simple one-dimensional (1-D) calculations usually isotropically add or subtract the magnetic pressure to the total pressure, which can generate a very large (but largely, due to the approximation involved, artificial) effect for stellar masses. However, for a large magnetic field, a general 2D treatment is necessary to take into account the anisotropic magnetic pressure.

The magnetic field is along the z-axis in the rest frame of the fluid, where the energy and pressure are given by

$$
\begin{array}{r}
\varepsilon=\varepsilon_{m}+\frac{B^{2}}{8 \pi}, \\
P_{\perp}=P_{m}-M B+\frac{B^{2}}{8 \pi}, \\
P_{\|}=P_{m}-\frac{B^{2}}{8 \pi},
\end{array}
$$


where $\varepsilon$ is the total energy density which is the sum of the matter energy density $\varepsilon_{m}$ and magnetic stress $\frac{B^{2}}{8 \pi}, P_{\perp}$ and $P_{\|}$are the perpendicular and parallel components of the total pressure with respect to the magnetic axis, $P_{m}$ is the matter pressure and $M B$ is the magnetization. It has been previously shown $[21,22,23]$ that the effect of landau quantization in the EoS is not significant for reasonable magnetic fields (although the magnetic field modifies significantly the central value of the EoS and population of the star [13]). The effect from magnetization can also as an approximation, be neglected. Therefore, the above equation can be written as

$$
\begin{gathered}
\varepsilon=\varepsilon_{m}+\frac{B^{2}}{8 \pi}, \\
P_{\perp}=P_{m}+\frac{B^{2}}{8 \pi}, \\
P_{\|}=P_{m}-\frac{B^{2}}{8 \pi} .
\end{gathered}
$$

The pressure can be conveniently described as

$$
\begin{array}{r}
P=P_{m} \pm \frac{B^{2}}{8 \pi}, \\
P=P_{m}+\frac{B^{2}}{8 \pi}\left(1-2 \cos ^{2} \theta\right),
\end{array}
$$

where, $\theta$ is the polar angle with respect to the magnetic field. Expanding it in terms of spherical harmonics

$$
\begin{array}{r}
P=P_{m}+\frac{B^{2}}{8 \pi}\left[\frac{1}{3}-\frac{4}{3} P_{2}(\cos \theta)\right], \\
P=P_{m}+\left[p_{0}+p_{2} P_{2}(\cos \theta)\right],
\end{array}
$$

where $p_{0}=\frac{B^{2}}{3.8 \pi}$ is the monopole contribution and $p_{2}=-\frac{4 B^{2}}{3.8 \pi}$ the quadrupole contribution.

The metric describing such magnetars can also be expanded in terms of multipole expansion. Limiting the expansion till quadrupole term $[14,24,25,26,27]$ gives

$$
\begin{array}{r}
d s^{2}=-e^{v(r)}\left[1+2\left(h_{0}(r)+h_{2}(r) P_{2}(\cos \theta)\right)\right] d t^{2} \\
+e^{\lambda(r)}\left[1+\frac{e^{\lambda(r)}}{r}\left(m_{0}(r)+m_{2}(r) P_{2}(\cos \theta)\right)\right] d r^{2} \\
+r^{2}\left[1+2 k_{2}(r) P_{2}(\cos \theta)\right]\left(d \theta^{2}+\sin ^{2} \theta d \phi^{2}\right),
\end{array}
$$

where $h_{0}, h_{2}, m_{0}, m_{2}, k_{2}$ are the corrections up to second order. 
Using the above equation in the Einstein equations and solving them, we get

$$
\begin{array}{r}
\frac{d m_{0}}{d r}=4 \pi r^{2} p_{0}, \\
\frac{d h_{0}}{d r}=4 \pi r e^{\lambda} p_{0}+\frac{1}{r} \frac{d v}{d r} e^{\lambda} m_{0}+\frac{1}{r^{2}} e^{\lambda} m_{0}, \\
\frac{d h_{2}}{d r}+\frac{d k_{2}}{d r}=h_{2}\left(\frac{1}{r}-\frac{\frac{d v}{d r}}{2}\right)+\frac{e^{\lambda}}{r} m_{2}\left(\frac{1}{r}+\frac{d v d r}{2}\right), \\
h_{2}+\frac{e^{\lambda}}{r} m_{2}=0, \\
\frac{d h_{2}}{d r}+\frac{d k_{2}}{d r}+\frac{1}{2} r \frac{d v}{d r} \frac{d k_{2}}{d r}=4 \pi r e^{\lambda} p_{2}+\frac{1}{r^{2}} e^{\lambda} m_{2} \\
+\frac{1}{r} \frac{d v}{d r} e^{\lambda} m_{2}+\frac{3 / r^{\lambda}}{e} h_{2}+\frac{2}{r} e^{\lambda} k_{2} .
\end{array}
$$

The total momentum conservation gives

$$
\begin{array}{r}
\frac{d p_{0}}{d r}=-\frac{d v}{d r} p_{0}-(\varepsilon+P) \frac{d h_{0}}{d r}, \\
p_{2}=-(\varepsilon+P) h_{2}, \\
\frac{d p_{2}}{d r}=-\frac{d \nu}{d r} p_{2}-(\varepsilon+P) \frac{d h_{2}}{d r} .
\end{array}
$$

Simple algebra gives the fields in terms of known quantities

$$
\begin{aligned}
\frac{d k_{2}}{d r} & =\frac{2 p_{2} \frac{d v}{d r}+\frac{d p_{2}}{d r}}{\varepsilon+P} \\
\frac{d h_{2}}{d r} & =\frac{-p_{2} \frac{d v}{d r}-\frac{d p_{2}}{d r}}{\varepsilon+P} .
\end{aligned}
$$

With known values of $p_{0}$ and $p_{2}$, we can calculate the correction terms.

The additional mass due to the magnetic field is given by $\delta M \equiv m_{0}$. Therefore, the total star mass is the sum of the mass due to matter $\left(M_{0}\right)$ and mass due to magnetic field

$$
M=M_{0}+\delta M .
$$

The star takes the shape of an oblate spheroid due to deformation induced by the non-isotropic magnetic pressure. The equatorial radius expands and the polar radius is compressed giving the star its deformed shape. The polar and equatorial radius are defined as

$$
\begin{array}{r}
R_{e}=R+\xi_{0}(R)-\frac{1}{2}\left(\xi_{2}(R)+r k_{2}\right), \\
R_{p}=R+\xi_{0}(R)+\left(\xi_{2}(R)+r k_{2}\right),
\end{array}
$$

where $R$ is the radius of the spherical star. $\xi_{0}$ and $\xi_{2}$ are defined as

$$
\begin{aligned}
& \xi_{0}(r)=\frac{r(r-2 G m(r))}{G\left(4 \pi r^{3} P_{m}+m(r)\right)} p_{0}^{*}, \\
& \xi_{2}(r)=\frac{r(r-2 G m(r))}{G\left(4 \pi r^{3} P_{m}+m(r)\right)} p_{2}^{*},
\end{aligned}
$$


with $p_{0}^{*}$ and $p_{2}^{*}$ given by

$$
\begin{aligned}
& p_{0}^{*}=p_{0} / \frac{B^{2}}{8 \pi}, \\
& p_{2}^{*}=p_{2} / \frac{B^{2}}{8 \pi} .
\end{aligned}
$$

With some given value of magnetic field and initial matter perturbation, one can calculate the correction terms and thereby determine the excess mass and star deformation. Following previous calculations $[17,18,20,21,22,28]$, we assume a density dependent magnetic field parameterized by

$$
B\left(n_{b}\right)=B_{s}+B_{0}\left\{1-e^{-\alpha\left(\frac{n_{b}}{n_{0}}\right)^{\gamma}}\right\} .
$$

With asymptotic magnetic field value $B_{0}=4 \times 10^{18} \mathrm{G}$, the maximum value of the central magnetic field we are considering is $B_{c}=1.75 \times 10^{18} \mathrm{G}$. For such field values, the magnetic pressure and energy are well below matter counterparts so that the perturbation calculations are justified. The surface magnetic field is fixed at $10^{15} \mathrm{G}$ and we assume $\alpha=0.01$ and $\gamma=2$. We are also using two sets of EOS (TM1 and NL3) [29, 30].

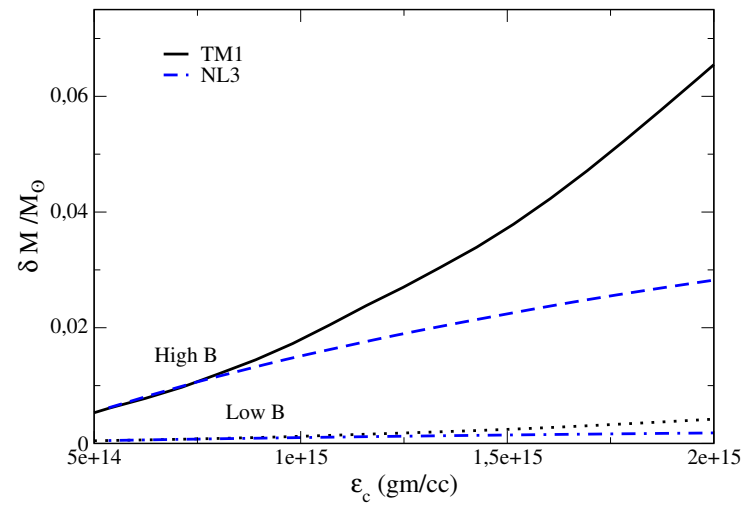

(a)

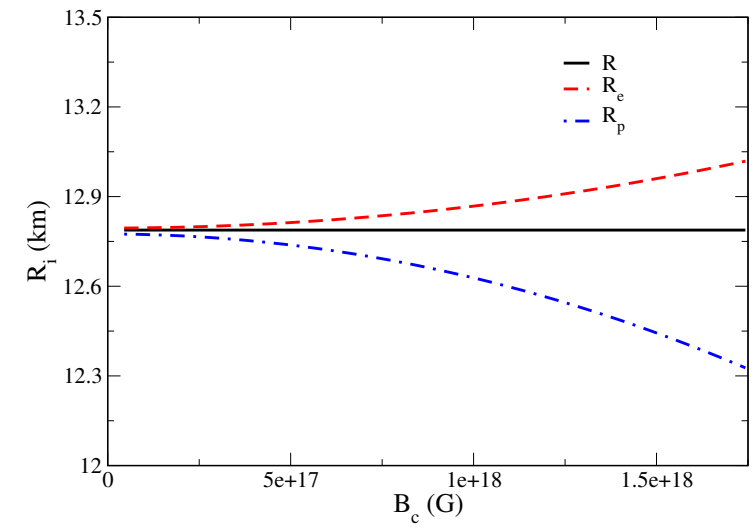

(b)

Figure 1: a) $\delta M$ as a function of central energy density $\varepsilon_{c}$ for two fixed central magnetic fields $B_{c}$. Curves are plotted for two different EoS's (TM1 and NL3). b) Different radial distances (including equatorial and polar radius) are plotted as a function of $B_{c}$.

Fig. 1a shows the additional mass due to magnetic field as a function of central energy density. As the central energy density increases, the magnetic field also increases (density dependent field) which in turn increases the additional mass. Two sets of curves are plotted, one with $B_{c}=1.75 \times$ $10^{18} \mathrm{G}$ and the other with $B_{c}=4.38 \times 10^{17} \mathrm{G}$. For the higher field strength, the excess mass is of the order of few percent (maximum for TM1 parametrization with $3-4 \%$ ) and, for the lower field, the excess mass is one order of magnitude lower. Fig. $1 \mathrm{~b}$ shows the increase of $R_{e}$ and the shrinking of $R_{p}$ in comparison to the spherical radius (shown for TM1 parametrization). The curves are plotted as a function central magnetic field. As the field increases, $R_{e}$ becomes much larger and $R_{p}$ becomes much smaller, giving the star a more oblate shape.

Next, we show the mass-radius (M-R) curve for the star with TM1 parametrization (Fig. 2). In Fig. 2a we compare the three sets of mass sequence. One for non-rotating non-magnetic stars, 
one with maximum rotational effect and one with maximum central magnetic field. The maximum rotational curve is inspired as the fastest rotating pulsar observed with rotational frequency of 716 Hz. The additional mass from both effects (rotation and magnetic field) are comparable, whereas the star's deformation is very different. The deformation due to rotation is quite large $\left(R_{e}\right.$ increases by about $500 \mathrm{~m})$ whereas the deformation due to magnetic field is small $\left(R_{e}\right.$ increases by $50-100$ $\mathrm{m})$. This can be simply understood; the rotational effect is very pronounced at the outer region of the star where the rotational velocity is maximum. The star mostly deforms in the outer regions where the density is very small compared to the star core. For magnetic field, the surface value is 3 order of magnitude lower than the central value and, therefore, the effect is very small in the outer region and the deformation is small.

In Fig. $2 b$ we find that as we increase the magnetic field, the mass of the star also increases. However, after some limiting value of the central field $B_{c}=2.6 \times 10^{18} \mathrm{G}$ there is no maximum mass and the mass of the star goes on increasing as the radius decreases. With such values of the magnetic field, the matter and magnetic pressures becomes comparable and the additional mass grows. This, on one hand, hints a limit of the magnetic field that can be present in the star within this description, whereas, on the other hand, it can signify that the star may be going towards a singularity.

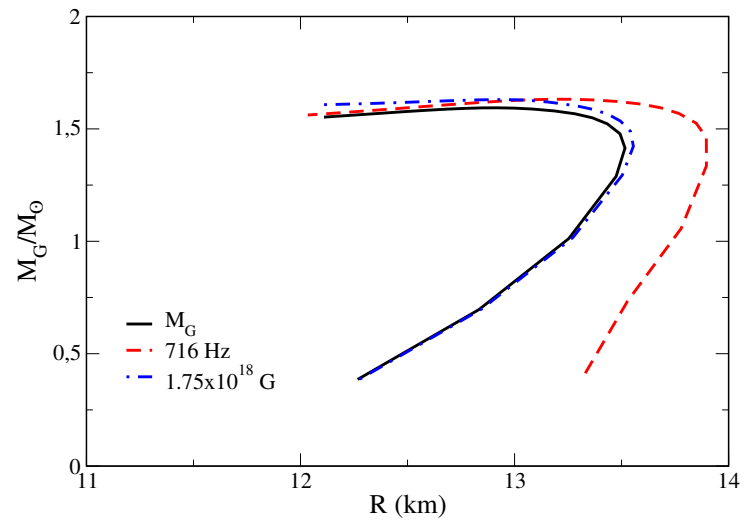

(a)

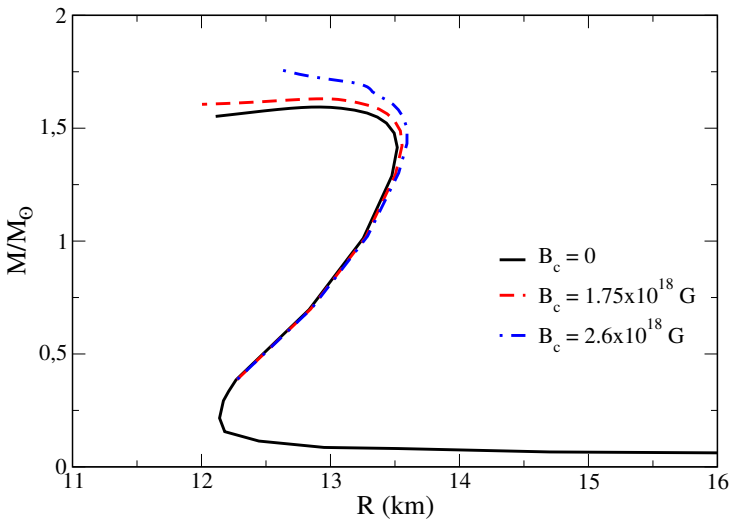

(b)

Figure 2: a) Star sequences are compared with magnetic and rotational effects with respect to a static nonmagnetic star. b) Star sequence with increasing magnetic fields are shown. For a strong enough magnetic field, the star has no maximum mass.

\section{Gravitational waves}

Magnetars are also strong candidates for continuous gravitational wave $(\mathrm{GW})$ emission. An uniformly rotating axisymmetric body does not emit gravitational waves, but an asymmetric body does. There are three classes of emission mechanisms of continuous GW from a spinning NS, (i) non-axisymmetric distortion of NS, (ii) unstable oscillation modes of the fluid part of the star and (iii) free precession. The presence of strong magnetic fields in magnetars can result in the emission of GW from mechanism (i) and (iii). If the rotation axis and the magnetic axis of the star are not aligned (which is usually the case for pulsars) the star will deviate from axisymmetry. It would also have some precession, that is the NS would wobble around the rotation axis as the rotation axis and the body symmetry axis would also differ. 
The amplitude of the GW signal can be written as [26, 31]

$$
h_{0}=\frac{4 G}{d c^{4}} \Omega^{2} I \varepsilon \sin \alpha,
$$

where $d$ is the distance of the star, $c$ the speed of light, $\Omega$ the rotational velocity, $I$ the moment of inertia, $\varepsilon$ the ellipticity and $\alpha$ the angle between the rotation and magnetic axis.

In the previous section, we have calculated the star's deformation which would give the ellipticity. With such values, one can make a crude estimate of gravitational waves (GW) coming from them. For the magnetar SGR $1900+14\left(B_{s}=7 \times 10^{14} \mathrm{G}\right)$, with mass of 1.5 solar mass, period of 5 sec and $d$ of $12.5 \mathrm{kpc}$, the amplitude of GW $h_{0}$ is $2.41 \times 10^{-28} \sin \alpha$. A similar estimate with SGR $10501+4516$ (closer to us, $d=2 \mathrm{kpc}$ ) gives $h_{0}=4.65 \times 10^{-28} \sin \alpha$ and AXP 1E 1841-045 leads to $h_{0}=4.54 \times 10^{-29} \sin \alpha$. With current capabilities of the VIRGO interferometric detector for a frequency of $30 \mathrm{~Hz}$ [32], the minimum amplitude detectable is of the order of $10^{-26}$, even with three years of data integration. Therefore, unfortunately, GW coming from such magnetars are not likely to be detected in the near future.

\section{Rho-condensation}

The condensation of pions in neutron stars was first suggested by Migdal [33, 34]. Since then there has been numerous discussion about its impact on NS physics and their observable signatures. After Kaplan [35] suggested kaon condensation in NS's, the field became more interesting. At the core of the star where matter is very dense the electron chemical potential increases. When pion/kaon energy becomes equal to the electron chemical potential it becomes favorable for neutrons to decay into protons and pions/kaons, rather than protons and electrons, giving rise to the corresponding condensate. However, a generic problem for such condensate is that it makes the EoS softer and reduces the mass of the star, which is in conflict with recent discovery of massive pulsars with masses of about $2 M_{\odot}$.

The condensation of vector meson in strong magnetic field has already been studies in the context of heavy-ion physics $[36,37,38]$. The simple idea is that higher-spin bosons interact strongly with the magnetic field leading to condensation. For heavy-ion physics, the mass of such vector meson has to me negative for condensation to happen, whereas for NS physics the mass of negative vector meson only has to drop below Fermi energy of electrons. The best candidate for such condensation in NS's is $\rho^{-}$meson.

For simplicity, we consider the GM3 [39] model for the EoS of NS's. The magnetic field makes the charged particles landau quantized perpendicular to the field [21]. The energy levels of the $\rho^{-}$are given by

$$
E_{\rho}=\sqrt{p_{z}^{2}+m_{\rho}^{2}+\left(2 n-g_{B} S_{z}+1\right) e B},
$$

Thus, the lowest Landau level at zero momentum with $g_{B}=2$ and $S_{z}= \pm 1$ has an energy of

$$
E_{\rho}^{2}=m_{\rho}^{2}-e B
$$

At the center of NS, where density is high, the hadronic masses are scaled [40, 41]. In the present calculation, we assume a simple density (field) dependent rho mass coupled to scalar $\sigma$ field:

$$
m_{\rho}^{*}=m_{\rho}-g \sigma
$$


with a free parameter $g$. Thus, the lowest-lying $\rho^{-}$state [42] is given by

$$
\left(E^{*}\right)_{\rho}=\sqrt{\left(m^{*}\right)_{\rho}^{2}-e B} .
$$

The $\sigma$ dependent $\rho^{-}$mass modifies the iso-spin properties of the model. Therefore, we have to adjust the nuclear-rho coupling and, for different choices of $g$, we keep the symmetry energy fixed at $32.5 \mathrm{MeV}$. The magnetic field is assumed to have strength of $7 \times 10^{18} \mathrm{G}$ which is on the higher side of the hypothetical accepted value possible in NS cores.

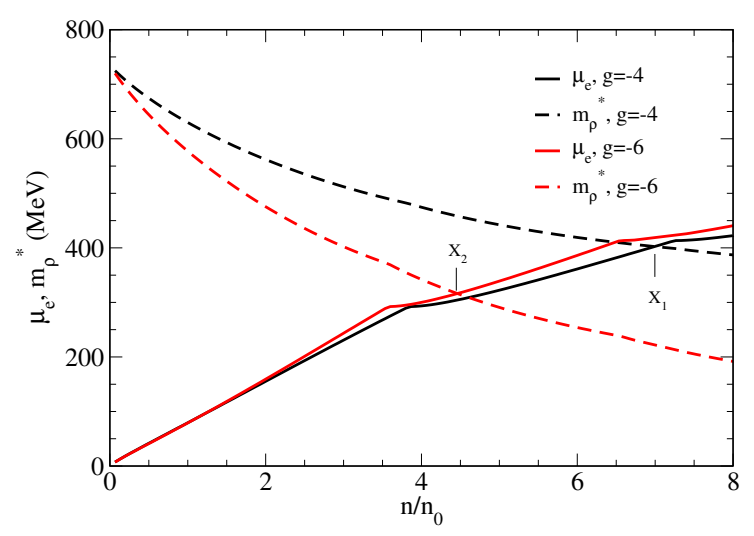

(a)

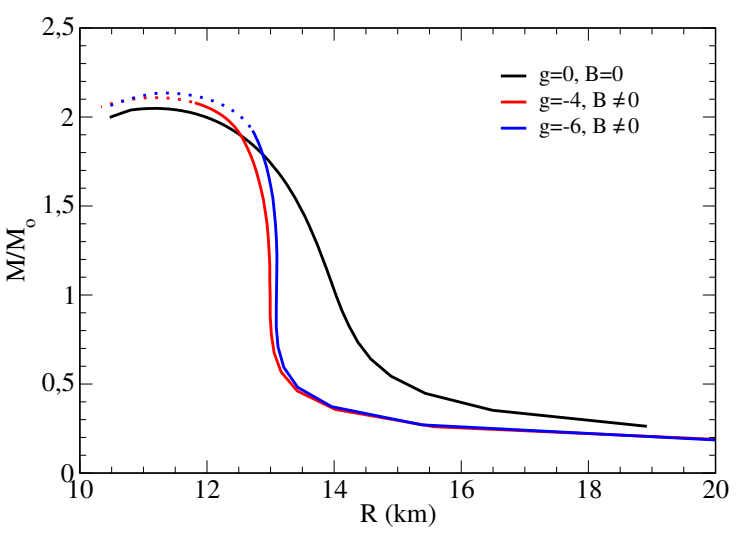

(b)

Figure 3: (a) The electron chemical potential (solid lines) and effective $\rho^{-}$mass (dashed lines) are plotted as functions of normalized density. Intersection points mark the density at which condensation happens. (b) Sequence of spherical stars (M-R curve) for the GM3 EoS. When both $g$ and $B$ are set to zero, there is no condensation. When $g$ and/or $B \neq 0$ the condensate appears. The dotted lines are stars which have some amount condensation in them.

The intersection points in Fig. 3a are denoted by $X_{1}$ and $X_{2}$. These points mark the initial normalized density where the condensation starts to set in. For a lower value of $g=-4$, the condensation stars at about 7 times nuclear density which is difficult if not impossible to be found in NS cores. For $g=-6$, the condensation starts at reasonable values and, for such stars, it is expected that their cores should have some amount of rho condensate in them. By increasing the value of $g$, the value of $m_{\rho}^{*}$ can be decreased and it is possible to achieve condensation at much lower densities. However, the lower bound of rho mass is provided by the experimental restriction on the value of symmetry energy slope $L[43,44,45]$. With such restriction, the rho condensation cannot start at values lower than 4 times nuclear density.

Solving the Tolman Oppenheimer Volkoff (TOV) equation with such EoS, we can determine how rho-condensation affects the star mass. In Fig. 3b, we plot the mass-radius curve for three different cases. With no magnetic or $\sigma$ effect on the mass there is no condensation. The other sequences have both effects. The dotted part of the curves represent stars which have some amount of rho-condensation at their cores. In the dotted region, the higher the mass, the higher the central density, thereby increasing the rho-condensation region at the star's core.

\section{Summary \& Discussion}

We have shown the effect of magnetic fields in different aspects of NS physics. In the first section, we have semi-analytically calculated the additional mass and star's deformation. Expanding 
the magnetic pressure and the perturbed metric in terms of spherical harmonics, we have perturbatively calculated the star's additional mass and deformed shape. For reasonable choices of magnetic field strengths, the effects were found to be less than $5 \%$.

The GW strength for known rotating magnetars (assuming that the rotation and magnetic axes are misaligned) is about $10^{-28}-10^{-29}$, which is of few order of magnitude lower that the expected amplitude to be detected by LIGO and VIRGO interferometric detectors. The situation can significantly improve by several orders of magnitude $\left(h_{0} \sim 10^{-23}-10^{-24}\right)$, for magnetars with higher rotational frequency. If they exists, their GW would be clearly in the detectability range of 2 nd generation interferometric detectors.

We have also discussed the possibility of negatively charged rho-meson condensation in magnetars. This can be achieved only if there is some sort of density (or field) effect on the rho mass. Note that such negatively charged rho-condensate stars (even not taking into account the extra mass allowed by the strong magnetic field) can still satisfy the new upper limit for neutron star masses.

Historically, NS's have proven to be excellent astrophysical laboratories for testing properties of matter under extreme conditions. The new generation of space-based X-ray and gamma-ray satellites are providing us with new observations and subsequently breakthrough discoveries $(\mathrm{kHz}$ quasi- periodic oscillations, bursting millisecond pulsars, X-ray super-bursts). On the other hand, with improved radio telescopes and interferometric techniques, the number of observed binary pulsars are continuously increasing. In recent time, multinational efforts have been made to build more sophisticated interferometric detectors for GW's. Thus, we are in the era of astrophysics, where NS's are going to play an important role in many aspects. The physics of high-energy heavyion collision explores one region in density and temperature of the strongly interacting matter (high temperature, low net density) while, as a complementary approach to the physics of strong interactions under extreme conditions, the other region (low temperature and high density) can only be tested by the signals coming from compact objects.

\section{References}

[1] Demorest, P., Pennucci, T., Ransom, S., Roberts, M., \& Hessels, J., Nature, 467, 1081 (2010)

[2] Antonidis, J., Freire, P. C. C., Wex, N. et. al., Science, 340, 448 (2013)

[3] Duncan, R. C., \& Thompson, C., AstroPhys. J., 392, L9 (1992)

[4] Thompson, C., \& Duncan, R. C., AstroPhys. J., 408, 194 (1993)

[5] Duncan, R. C., \& Thompson, C., Astrophys. J. 473, 322 (1992)

[6] Paczynski, B., Acta. Astron. 42, 145 (1992)

[7] Melatos. A., Astrophys J. Lett. 519, L77 (1999)

[8] Mallick, R., Schramm, S., Phys. Rev. C 89, 025801 (2014)

[9] Bocquet, M., Bonazzola, S., Gourgoulhon, E., \& Novak, J., Astron. \& AstroPhys., 301, 757 (1995)

[10] Cardall, C. Y., Prakash, M., \& Lattimer, J. M., AstroPhys. J., 554, 322 (2001)

[11] Potekhin, A. Y., \& Yakovlev, D. G., Phys. Rev. C 85, 039801 (2012)

[12] Manreza Paret, D., Perez Martinez, A., \& Horvath, J. E., ArXiv:1501.04619 (2015)

[13] Franzon, B., Dexheimer, V., \& Schramm, S., ArXiv:1508.04431 (2015)

[14] Chandrasekhar, S., and Fermi, E., AstroPhys. J. 118, 116 (1953)

[15] Bonazzola, S., Gourgoulhon, E., Salgado, M., and Marck, J. A., Astron. \& Astrophys. 278,421 (1993) 
[16] Ciolfi, R., Ferrari, V., Gualtieri, L., \& Pons, J. A., Mon. Not. Roy. Astron. Soc., 397, 913 (2009)

[17] Chakrabarty, S., Bandyopadhyay, D., \& Pal, S., Phys. Rev. Lett., 78, 2898 (1997)

[18] Bandyopadhyay, D., Chakrabarty, S., Dey, P., \& Pal, S., Phys. Rev. D, 58, 121301 (1998)

[19] Mallick, R., and Sinha, M., MNRAS 414, 2702 (2011)

[20] Rabhi, A., Providencia, C., \& Da Providencia, J. Da, J. Phys. G: Nucl. Part. Phys. 35, 125201 (2008)

[21] Dexheimer, V., Negreiros, R., \& Schramm, S., Eur. Phys. J. A, 48, 189 (2012)

[22] Sinha, M., Mukhopadhyay, B., \& Sedrakian, A., Nucl. Phys. A, 898, 43 (2013)

[23] Chatterjee, D., Elghozi, T., Novak, J., \& Oertel, M., MNRAS 447, 3785 (2015)

[24] Hartle, J. B., AstroPhys. J., 150, 1005 (1967)

[25] Hartle, J. B., \& Thorne, K. S., AstroPhys. J., 153, 807 (1968)

[26] Bonazzola, S., \& Gourgoulhon, E., Astron. \& AstroPhys., 312, 675 (1996)

[27] Konno, K., Obata, T., \& Kojima, Y., Astron. \& AstroPhys., 352, 211 (1999)

[28] Lopes, L. L., \& Menezes, D. P., Braz. J. Phys. 42, 428 (2012)

[29] Sugahara, Y., \& Toki, H., Nucl. Phys. A 579, 557 (1994)

[30] Shen, G., Horowitz, C. J., \& Teige, S., Phys. Rev. C 82, 045802 (2010)

[31] Ciolfi, R., Ferrari, V., \& Gualtieri, L., Mon. Not. Roy. Astron. Soc., 406, 2540 (2010)

[32] Bonazzola, S., \& Marck, J. A., Annu. Re. Nucl. Part. Sci., 45, 655 (1994)

[33] Migdal, A. B., Soviet Phys. JETP 61, 2209 (1971)

[34] Migdal, A. B., Phys. Lett. 45B, 448 (1973)

[35] Kaplan, D. B., \& Nelson, A. E., Phys. Lett. B 175, 57 (1986)

[36] Schramm, S., Mueller, B., \& Schramm, A. J., Mod. Phys. lett. A 7, 973 (1992)

[37] Huang, Mei, Zhuang, Peng-fei, \& Chao, Wei-qin, Phys. Rev. D 65, 076012 (2002)

[38] Chernodub, M. N., Phys. Rev. D 82, 085011 (2010)

[39] Glendenning, N. K., \& Moszkowski, Phys. Rev. Lett. 67, 2414 (1991)

[40] Voskresensky, D. N., Phys. Lett. B 392, 262 (1997)

[41] Kolomeitsev, E. E., \& Voskresensky, D. N., Nucl. Phys. A 759, 373 (2005)

[42] Mallick, R. \& Schramm, S., MNRAS 449, 1347 (2015)

[43] Chen, L. -W., Ko, C. M., \& Li, B. -A., Phys.Rev. C72, 064309 (2005)

[44] Tsang M. B., Stone, J. R., Camera, F., \& et al, Phys. Rev. C 86, 015803 (2012)

[45] Lattimer, J. M., \& Lim, Y., Astrophys. J. 771, 51 (2013) 